\title{
On the optimal object orientation in additive manufacturing
}

\author{
S. Pereira* \\ A. I. F. Vaz* \\ L. N. Vicente ${ }^{\dagger}$
}

May 16, 2018

\begin{abstract}
Additive manufacturing (AM) has emerged in the last decades as an alternative to traditional subtractive and forming manufacturing processes. AM is a process where $3 \mathrm{D}$ objects are built by adding material in consecutive layers. Typical AM is characterized by four processing stages: model orientation, creation of supports, slicing, and path planning. In this paper we will focus on how to use mathematical optimization to address some limitations that arise from the model orientation, creation of supports, and slicing processing stages.

Model orientation and creation of supports are usually related, since the best orientation of the object to be built can result in lower building time and lower need for creation of supports, leading to an improvement of the surface quality. Slicing comprises the object division by layers and the major difficulty is the staircase effect, which becomes more critical for objects with high slopes and curvatures, resulting in high roughness surfaces.

In this paper we show how to take advantage of a state-of-the-art optimization solver to optimize object building orientation and the need for supports generation in additive manufacturing, thus leading to better object surface accuracy and smoothness.
\end{abstract}

Keywords: Additive manufacturing; 3D printing; model orientation, supports generation, slicing

\section{Introduction}

Additive manufacturing (AM) has been used over the last decades with a hight acceptance in aeronautics and auto-mobile industries, in medical applications, and in the field of

\footnotetext{
*ALGORITMI Research Center, University of Minho, Campus of Gualtar, 4710-057 Braga, Portugal. ( sergiop@dps.uminho.pt, aivaz@dps.uminho.pt $\}$ ).

${ }^{\dagger}$ CMUC, Department of Mathematics, University of Coimbra, 3001-501 Coimbra, Portugal (lnv@mat.uc.pt).
} 
biomedical engineering [23]. Also known as 3D printing or layer-by-layer manufacturing (LM), additive manufacturing is a process where a specific object is produced using layerby-layer deposition of material [37]. Jin et al. [13] defines it as a group of layer-based joining processes that builds physical shapes and structures directly from virtual models. The first technique consists in converting the information of a CAD file into a stereolithography (STL) file [8]. STL is nothing else than an approximation (tessellation) of the CAD model in which the geometrical features of the 3D models are described by a mesh of triangles. Such a technique eases the way of dealing with the models and, therefore, STL became one of the most popular and widely accepted used file format in the LM industry [10].

Layer-by-layer manufacturing processes emerged as an alternative to the traditional subtractive and forming manufacturing processes. Contrary to LM, subtractive manufacturing consists in the use of techniques such as milling, sawing or water-jet cutting [31]. Using these manufacturing techniques can be difficult mostly in objects/solids where the geometry is complex. AM changed the paradigm of traditional manufacturing processes, being capable to deal with objects of high complexity and reducing environmental impact by efficient use of material $[4,5]$. Additionally, LM processes require very little human intervention and set-up time [18]. Several techniques for LM may be used, like, for example, directed energy deposition [16, 34, 9], 3D printing [24, 21, 30], laser sintering [41, 17], and STereoLithography $[7,3]$. 3D printing is one of the most wildly used techniques in the LM process supported by the large proliferation of inexpensive machines and the diversity of available software to drive those machines [25].

LM poses some challenges related to models surface quality. The stair-stepping effect is one of the major problems inherent to LM [22]. Other challenges pointed out to LM are the low deposition quality, largely related to the filling strategy (the path deposition length and the strategy itself) and the type of used material, as well as the poor surface finish of printed objects. These challenges pose difficulties to the dissemination of LM techniques [14].

Typically four planning stages must be considered in LM: initial orientation of the objects/parts being built, supports generation to ensure that overhanging features can be built without presenting major object deformations, slicing, and path planning [18]. This paper focus on the first three planning stages.

A properly selection of the initial object orientation is essential to reduce the supports generation need. Some authors claim that it can also reduce the building time of the desired objects. However, some objects may be impossible to build without the use of supporting structures.

The slicing task refers to a procedure in which planes are intersected with the model in order to determine contours defining where material will be deposited [18]. Over the last decades, different slicing strategies have been proposed for different LM techniques.

This paper contribution is the use of a state-of-the-art optimization solver to address the optimization of the final printed object surface, based on minimizing the staircase effect and the need of supports. Such an optimization will then simplify the slicing process and consequently improve the models surface accuracy.

This paper is structured as follows. In Section 2 it is presented an overview of the 
literature regarding supports generation, built objects orientation, and slicing. In Section 3, our improved optimization methodology is presented, consisting of the numerical solution of a non-differentiable non-linear bound-constrained optimization problem. In Section 4, we report some numerical results obtained with objects from the aeronautic industry. Section 5 contains some conclusions and prospects of future work.

\section{Overview of existing approaches}

Support generation and model orientation are two tasks of the LM process that can significantly influence the result of any built object. Often, both support generation and model orientation are dependent of each other, since only after model orientation it is possible to determine the overhanging parts of the model and thus the need or not of support generation. According to Kulkarni et al. [18], two types of supports can be considered: internal and external. While external supports are essential to support overhanging features, internal are used to support models parts with hollow surfaces. The need of supports must be minimized, since it leads to increasing costs of the manufacture objects due to the increase of building time and consumed material and to the decrease of surface quality in places where supports are built [18].

The manufacturing time of an object depends on its initial orientation as the number of slices to be considered varies with object orientation. Object orientation can improve the accuracy of the built object and reduce the number of generated supports, and consequently decrease the final building costs. Cheng et al. [2] presented a multi-objective optimization problem to determine the optimal object building orientation. Essential requirements pointed out by these authors to obtain the best object building orientation are: maximization of the number of perpendicular surfaces, maximization of the number of up-facing horizontal surfaces, maximization of the number of holes with their axes in the slicing direction, maximization of the area of the base surface, minimization of the number of slope surfaces, minimization of the total area of overhang surfaces, minimization of the total number of slices, and minimization of the height of required support structures. Richard et al. [33] consider the strength of the building objects as a measure of quality. Their objective function takes into account the object strength, the surface errors, the building time, and the volumetric supports.

In Hussein et al. [11], a new strategy to minimize the negative effects of supports in the manufacturing procedure is presented. These authors introduce a new design and manufacturing support characterized by its efficiency. Such a support has the form of a lattice structure which results in a very low volume, leading to a significant amount of materials savings and a reduction of the building time. Strano et al. [36] describes a new approach to minimize the need of support structures. They have developed a new algorithm that performs a two-step optimization procedure by firstly obtaining the best orientation that originates the minimum use of supports and then generating a cellular support structure using the computed orientation. This strategy leads to significant materials saving along with building time improvement. 
The slicing process in AM/LM also poses significant challenges. This process consists of cutting any 3D model into a set of slices with a certain thickness. Therefore, each slice is nothing else than a model layer represented in a two axes plane. The 3D model is then obtained by vertically incrementing each layer over a third axis with the step corresponding to the layer thickness. Slicing can be classified as direct when it takes place from a computed aided design (CAD) software or indirect when the object is represented as an approximation (usually in STL format, where the object is described as a mesh of triangles and their respective normal vectors). Over the last years different slicing strategies have been proposed in the literature. The bottlenecks of the LM process can be reduced by using appropriate slicing processes. According to Oropallo et al. [25] there are two main issues regarding the slicing process. One is the staircase effect due to the stacking of each layer and the other is what it is called the containment problem. These problems occur since different layers may fall inside or outside of the original objects contours as is schematically depicted in Figure 1 (in two dimensions for a better visualization), where the original object is represented by a disc and layers are represented by rectangles. The staircase effect results from the representation of curved objects by layers and the containment problem consists in representing the object by layers that are approximating the object from inside or outside.

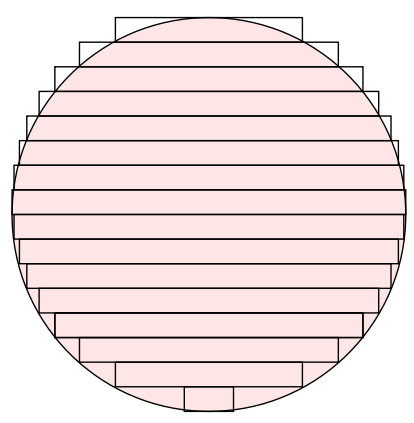

Figure 1: Staircase effect and containment problem.

Despite the method used for slicing (direct or indirect) there are two main different strategies concerning the slicing process. Slicing can be uniform and adaptive, the former is used for the construction of layers with the same thickness, and the later to construct layers with different thickness (adaptive). The adaptive layer thickness usually depends on the slope and curvature of the object: thicker slices are used for thicker slopes and large curvatures and thinner slices are used for thinner slopes or small curvatures. Adaptive slicing was firstly presented and addressed in Dolenc et al. [6], where it is presented a way to restrict the staircase effect. This is achieved by selecting a layers thickness given by the cusp height tolerance (meaning the measure between the slice vertex and the model surface).

Figure 2 depicts the cusp height to better understand how it can be used as a measurement of the quality of the built objects. A simple inspection of Figure 2 gives rise to Equation (1) below, which gives us the relation between the building angle $(\beta)$, the cusp 
height $(H c)$, and the layers thickness $(t)$. The object staircase effect can be measured by summing up all the cusp heights formed between every slice and mesh triangle.

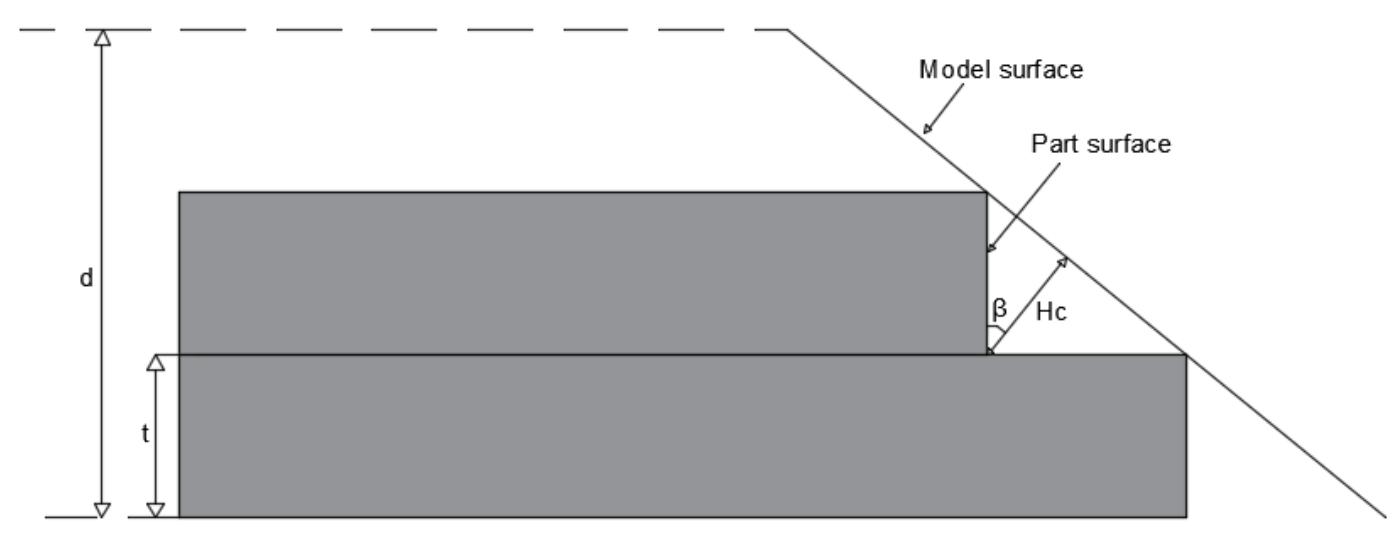

Figure 2: Cusp Height $(H c)$ representation.

$$
\cos (\beta)=\frac{H c}{t} \quad \Longleftrightarrow \quad \beta=\arccos \left(\frac{H c}{t}\right)
$$

Lower cusp heights promote a better approximation of the object contours, leading to lower layers thickness, and therefore a balance between the cusp height and the number of layers must be considered since the building time often depends on the number of layers. In Jung et al. [15], the cusp height is used to measure the quality between two consecutive layers. Wang et al. [40] developed a technique to reduce the manufacturing time of 3D printing using an adaptive slicing strategy to optimize slices thickness. Printed objects are evaluated by considered the cusp height as a measure of quality. The proposed technique consists in the division of the object in sub-parts, independently optimizing the slicing for each one. The results presented led to saves of $30-40 \%$ in the printing time. In Rianmora et al. [32], an adaptive direct slicing approach is addressed. This approach consists in the application of an image processing technique to determine appropriate thickness for each sliced layer and to recommend slicing positions. The obtained results were compared with different techniques using different cusp height values. Results show that the adaptive direct slicing approach leads to a lower number of layers with direct impact on the building time. Other works considering the cusp height as a quality measure can be seen in $[20,19,27,35]$. Jibin [12] introduces a tri-objective approach considering the building time as one objective and where the cusp height is used to formulate two additional objective functions.

Object surface roughness can be measured by looking at the $R a$ value. The $R a$ value is computed by considering an experimentally obtained confidence interval for the roughness. The $R a$ can be obtained by using, e.g., Equation (2) and was firstly addressed in [26, 28].

$$
R a=(a \text { to } b) \frac{t}{\cos \beta},
$$


where ( $a$ to $b)$ is the confidence interval, $t$ is the layer thickness, and $\beta$ is the angle between the building direction vector and the normal vector. The $R a$ quality measure is also used in [29]. In this study a multi-criteria genetic algorithm was used in order to determine the optimal object deposition orientations. The two objectives used are the surface roughness and the building time. Singhal et al. [35] developed an adaptive slicing strategy using the surface roughness of objects, and this procedure was considered as starting point for the work in [1].

\section{Model description}

Jibin [12] introduced a new multi-objective optimization strategy to simultaneously minimize the staircase effect, the need for supports, and the building time. This author presented some numerical results for an object, to which a genetic algorithm for multi-objective optimization was then applied. However, as we will see in this section, we only need to consider a single objective when building symmetric objects. This simplification will then allow the application of a state-of-the-art solver from derivative-free global optimization.

While in some additive manufacturing processes the layer thickness can vary among layers, we consider it to be constant in the slicing process since this is the case in the applications of interest to us. Taking into account the cusp definition presented in Equation (1) one can compute the staircase effect $(S E)$ considering the total cusp height by summing up all the individual cusp contributions, leading to the following equation:

$$
S E=\sum_{j} \frac{t^{2}\left|d . n_{j}\right| A_{j}}{2},
$$

where $t$ is the (constant) layers height, $d$ is a normalized (i.e., $\|d\|=1$ ) slicing direction, $n_{j}$ is a normalized mesh triangle $j$ normal vector, and $A_{j}$ is the mesh triangle $j$ area.

The need for supports can also be measured by considering the cusp height, but in this case taking it only when the facets are facing down. While many AM processes can manufacture overhanging surfaces with an inclination near to 45 degrees, in our case, and without loss of generality, we consider all the facets facing down (corresponding to the case where $d . n_{j}$ is negative in (5) below). Therefore the need for supports can be measured by the following equation representing the support area $(S A)$ :

$$
S A=\sum_{j} A_{j}\left|d \cdot n_{j}\right| \delta
$$

where

$$
\delta= \begin{cases}1, & d \cdot n_{j}<0 \\ 0, & d \cdot n_{j}>0\end{cases}
$$

An approximation to the building time $(B T)$ may be obtained by computing the object height along the slicing direction, leading to the following equation:

$$
B T=\max \left(d \cdot v_{1}, d . v_{2}, \ldots, d . v_{n}\right)-\min \left(d \cdot v_{1}, d . v_{2}, \ldots, d \cdot v_{n}\right),
$$


where $v_{i}, i=1, \ldots, n$, are the mesh triangles vertices.

However, the objects in which we are interested exhibit some regularity in the sense that the number and area of triangles leading to $d . n_{j}>0$ are the same of the ones leading to $d . n_{j}<0$, which then makes the $S E$ and $S A$ objective functions defined by equations (3) and (4) non conflicting. Also, $S E$ and $S A$ should not account for all mesh triangles, since the cusp is not well defined when $\left|d \cdot n_{j}\right|=1$ and there is no need to build support at mesh triangles in the printing table base where $d . n_{j}=-1$.

The building time will not be considered in the present work, since the simplification of the true building time given by Equation (6) is not appropriate for the type of objects of interest to us. Future work includes the definition of a proper and accurate building time measure, taking into consideration the printer characteristics.

We will therefore use the following objective functions for numerical testing:

$$
\widehat{S E}=\frac{t^{2}}{2} \sum_{j}\left\{\begin{array}{c}
\left|d . n_{j}\right| A_{j}, \text { if }\left|d . n_{j}\right| \neq 1 \\
0 \text { otherwise. }
\end{array}\right.
$$

$$
\widehat{S A}=\sum_{j}\left\{\begin{array}{c}
A_{j}\left|d \cdot n_{j}\right| \delta, \text { if } d \cdot n_{j} \neq-1 \text { and triangle } j \text { is not at the printing table base } \\
0 \text { otherwise. }
\end{array}\right.
$$

We provide numerical results using both objective functions independently, thus showing that they are not conflicting in our case and lead to the same optimal design.

\section{Numerical results}

We consider the bound-constrained optimization problem

$$
\min _{\theta \in[0,180]^{2}} f(\theta),
$$

where $\theta=\left(\theta_{x}, \theta_{y}\right) \in[0,180]^{2}$ are the object rotation angles (in degrees) along the $x$ and $y$ axes. Recall that this is equivalent to compute a slicing normalized direction $d$.

The numerical results reported here were performed using the three different objects displayed in Figure 3. The "Humanoid" is included due to its simplicity and the other two objects ("T-Duct" and "Duct") are applications of 3D printing to the aerospace industry. Each object has a specific degree of complexity indexed by the number of triangles (facets) used to compose the object. Different object length scales can be used in the proposed geometries having no relevance to the physics of the process. The only concern with object length is the $3 \mathrm{D}$ printer physical limitations. Table 1 presents the number of triangles used to describe each object and object size.

As already stated, we are looking for a global minimum of problem (7) and thus we have selected one of the state-of-the-art solvers for global derivative-free optimization subject 


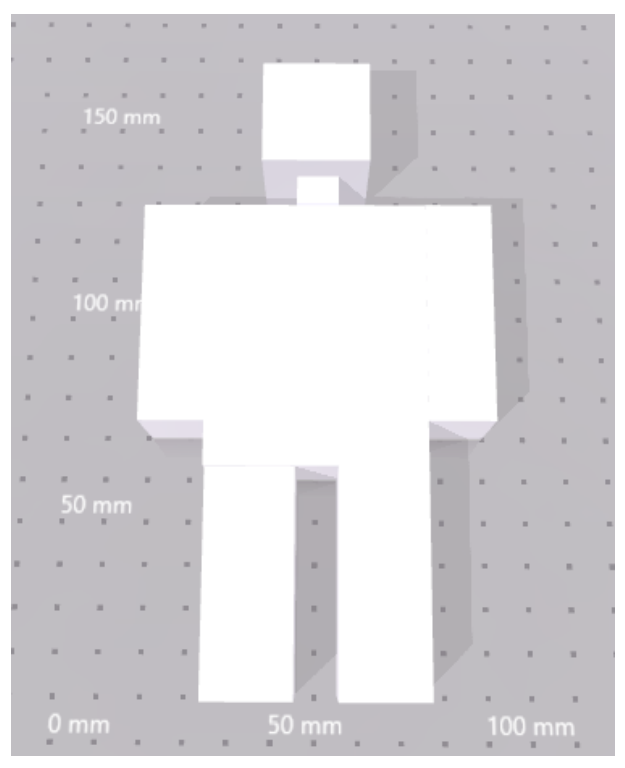

(a)

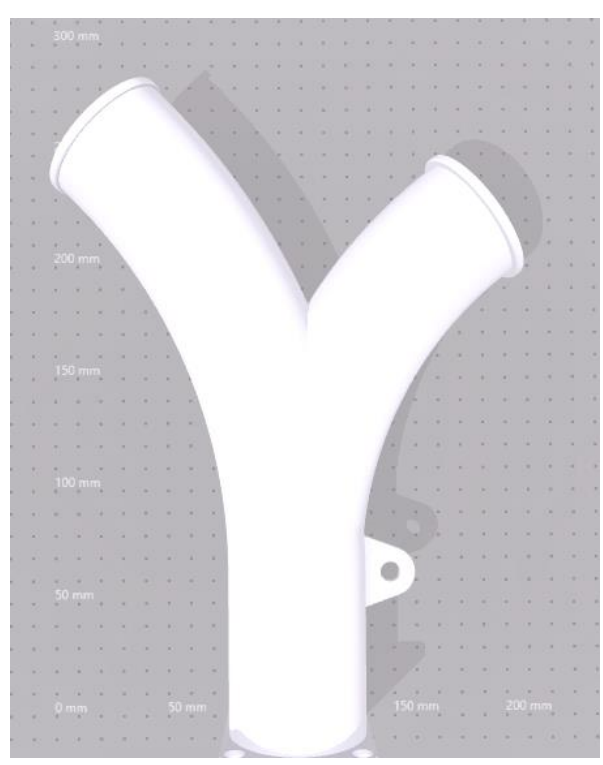

(b)

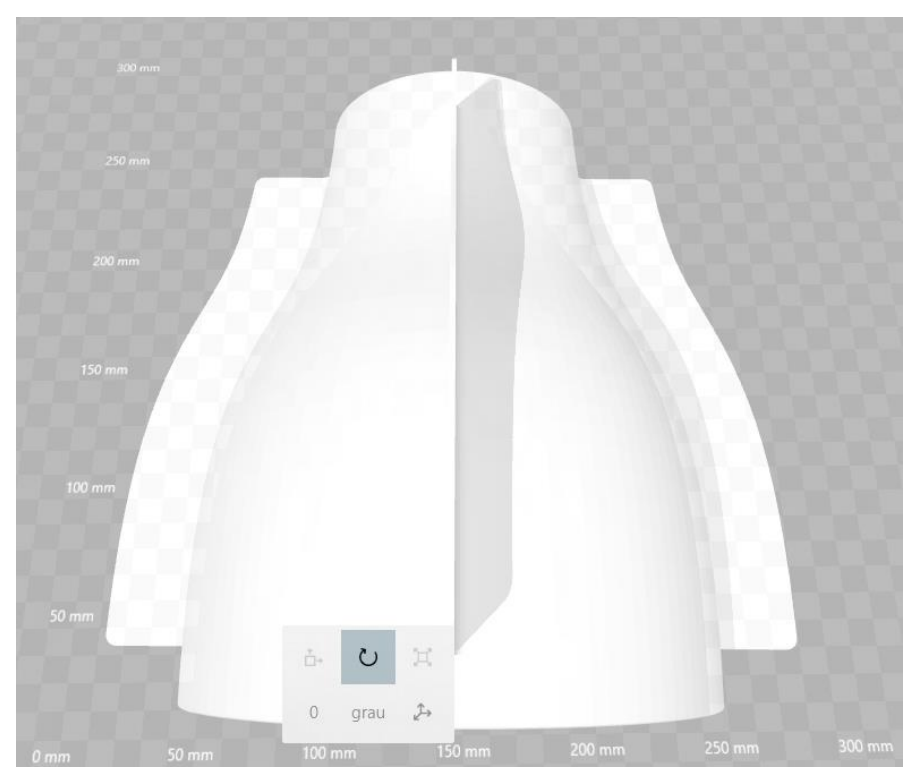

(c)

Figure 3: Objects used for simulation. 
Table 1: Object complexity.

\begin{tabular}{c|c|c|c}
\hline & Humanoid & T-Duct & Duct \\
\hline $\mathrm{N}^{o}$ of triangles & 96 & 19686 & 28656 \\
Object size $(\mathrm{mm})$ & $79.95 \times 29.14 \times 144.48$ & $191.15 \times 60 \times 270.41$ & $316.05 \times 316.03 \times 285.17$ \\
\hline
\end{tabular}

to simple bounds on the variables (PSwarm [38, 39], available at www.norg.uminho.pt/ aivaz/pswarm). We depict the objective function landscapes in Figure 4 for the selected objects. We can easily check that $S E$ and $S A$ objective functions are not confliting. The numerical results are presented in Tables 2-4, where we report the final solution obtained as well as its objective value. We also report the total CPU time (on an Intel(R) Core(TM) i7-7500U CPU $2.70 \mathrm{GHz}$ 12GB RAM), the number of iterations taken by PSwarm, and the number of objective function evaluations needed to obtain the solution.

Table 2: Numerical results for the SE objective function.

\begin{tabular}{c|c|c|c|c|c}
\hline & $\begin{array}{c}\text { Problem } \\
\text { solution }\end{array}$ & $\begin{array}{c}\text { Objective } \\
\text { function value }\end{array}$ & Time & \# Iterations & $\begin{array}{c}\text { \# Function } \\
\text { evaluations }\end{array}$ \\
\hline $\begin{array}{c}\text { Duct } \\
\text { Humanoid }\end{array}$ & {$[90,0]$} & $8.143735 \exp +3$ & $1 m 31 s$ & 43 & 453 \\
T-Duct & {$[90,180]$} & $9.325598 \exp -7$ & $0 s$ & 88 & 478 \\
\hline
\end{tabular}

Table 3: Numerical results for the SA objective function.

\begin{tabular}{c|c|c|c|c|c}
\hline & $\begin{array}{c}\text { Problem } \\
\text { solution }\end{array}$ & $\begin{array}{c}\text { Objective } \\
\text { function value }\end{array}$ & Time & \# Iterations & $\begin{array}{c}\text { \# Function } \\
\text { evaluations }\end{array}$ \\
\hline Duct & {$[90,0]$} & $3.289783 \exp +4$ & $1 m 9 s$ & 42 & 395 \\
Humanoid & {$[0,90]$} & $6.519794 \exp +1$ & $0 s$ & 88 & 644 \\
T-Duct & {$[90,0]$} & $1.470386 \exp +4$ & $57 s$ & 44 & 442 \\
\hline
\end{tabular}

Table 4: Numerical results for the BT objective function.

\begin{tabular}{c|c|c|c|c|c}
\hline & $\begin{array}{c}\text { Problem } \\
\text { solution }\end{array}$ & $\begin{array}{c}\text { Objective } \\
\text { function value }\end{array}$ & Time & \# Iterations & $\begin{array}{c}\text { \# Function } \\
\text { evaluations }\end{array}$ \\
\hline Duct & {$[180,50]$} & $2.359555 \exp +2$ & $1 m 29 s$ & 53 & 454 \\
Humanoid & {$[90,0]$} & $2.869000 \exp +0$ & $0 s$ & 88 & 478 \\
T-Duct & {$[180,180]$} & $6.000000 \exp +1$ & $43 s$ & 36 & 357 \\
\hline
\end{tabular}




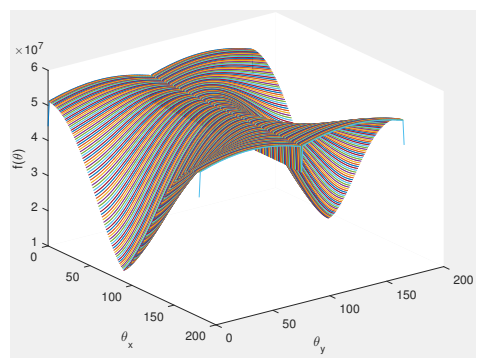

(a) Duct SE function

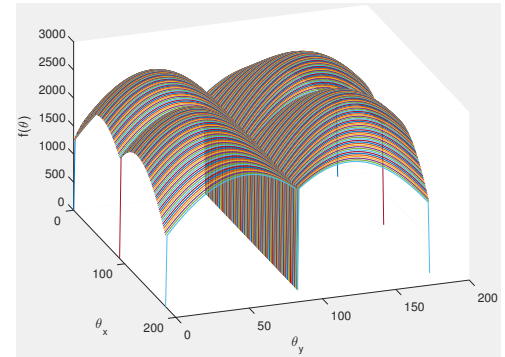

(d) Humanoid SE function

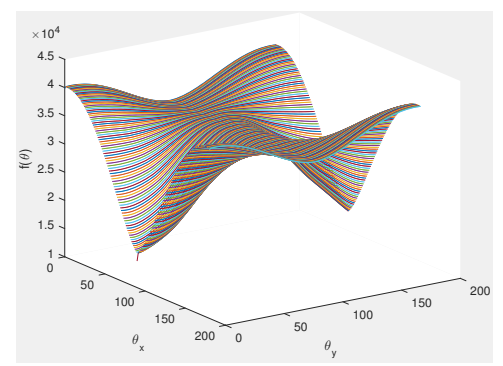

(g) T-Duct SE function

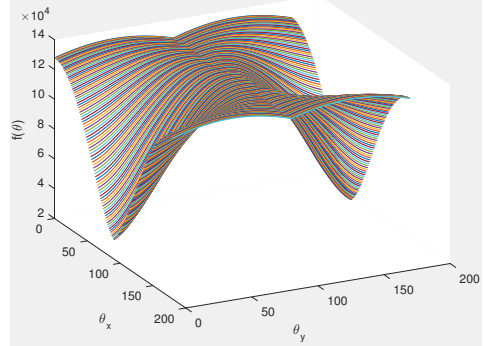

(b) Duct SA function

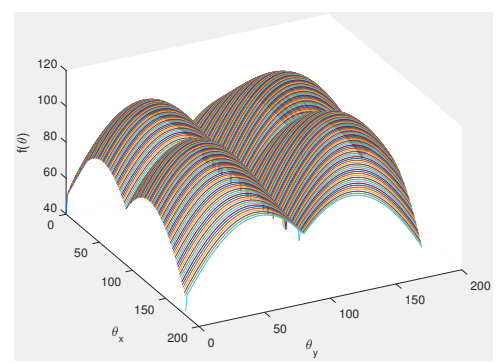

(e) Humanoid SA function

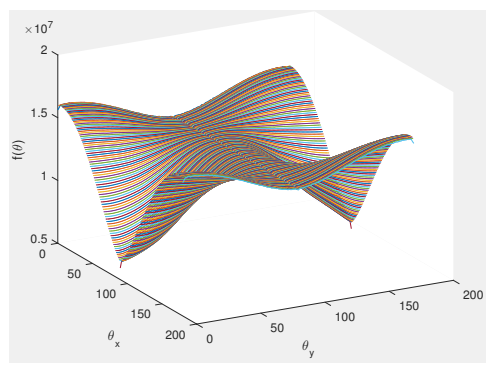

(h) T-Duct SA function

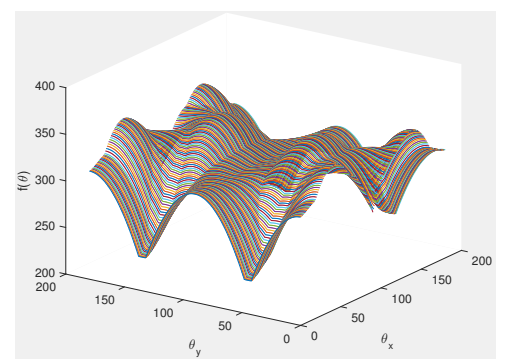

(c) Duct BT function

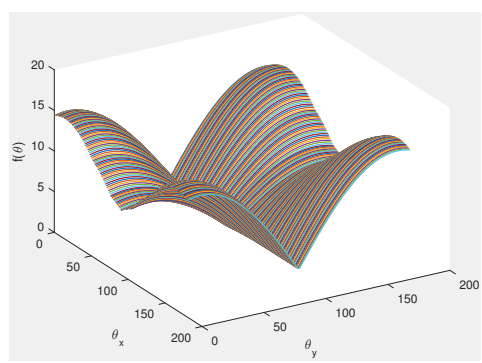

(f) Humanoid BT function

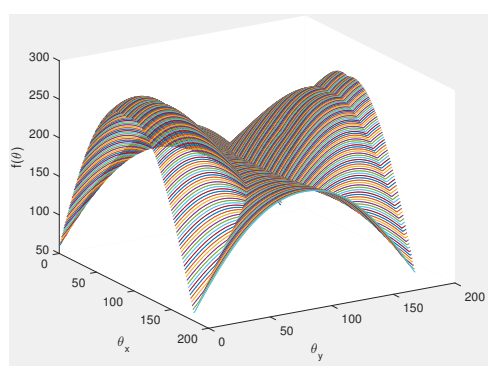

(i) T-Duct BT function

Figure 4: Objects landscape for each objective function. 
A closer look to Tables 2-3 allows us to conclude that, while $S E$ and $S A$ take different objective function values, they lead to the same optimal solution $\theta=\left(\theta_{x}, \theta_{y}\right)=[90,0]$ for the "Duct" object, i.e., a rotation along the $x$ axis of 90 degrees. While the solutions for the object "T-Duct" are different for these objective functions they represent the same optimal solution in terms of slicing (staircase and supports), since a rotation of 180 degrees around the $y$ axis has no impact on the final object building (see Figure 5 where both solutions slicing are depicted). These results confirm the non conflicting nature of the $S E$ and $S A$ objective functions for regular objects.

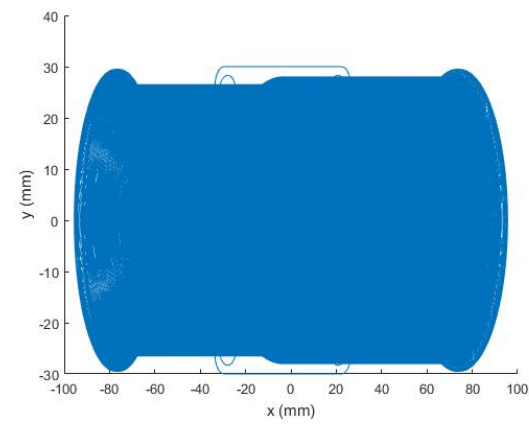

(a) With the SE function

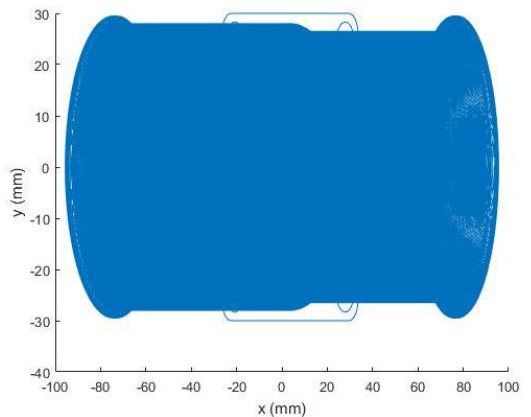

(b) With the SA function

Figure 5: T-Duct final optimal orientation.

Contrary to both "Duct" and "T-Duct" objects, the "Humanoid" object leads to different results when using the $S A$ or the $S E$ objective functions. As it can be observed in Figure 3, the "Humanoid" is not symmetrical, since it has one leg thinner than the other. This leads to different solutions, $\theta=\left(\theta_{x}, \theta_{y}\right)=[90,0]$ and $\theta=\left(\theta_{x}, \theta_{y}\right)=[0,90]$, for the objective functions $S E$ and $S A$, respectively. Figure 6 depicts the slicing obtained after the optimal rotation, confirming the usefulness of considering each of these two objective functions individually. When using the objective function $S E$ the staircase effect will be minimized discarding any concern with the support, leading to suspended legs, while using the objective function $S A$ the support area will be minimized and the object is built in a position parallel to the $x y$ plane.

Numerical results support the need to use global optimization to achieve a satisfactory approximate solution for the optimization problem (7), due to the existence of many local minimizers and the extensive presence of non-differentiability, thus excluding the possibility to use gradient or Newton type methods.

Table 4 presents the results corresponding to the minimization of the building time. While this objective function is not of a major interest to our work we include it for a matter of completeness. The time to build an object is a function of its height along the slicing direction, which in our case corresponds to the height along the $z$ axis. In fact, Equation (6) accounts for the movement of the extruder head/support table along the slicing direction. Optimal solutions for the "T-Duct" and "Humanoid" cases correspond to the objects being parallel to the $x y$ axes while the "Duct" optimal solution is described 


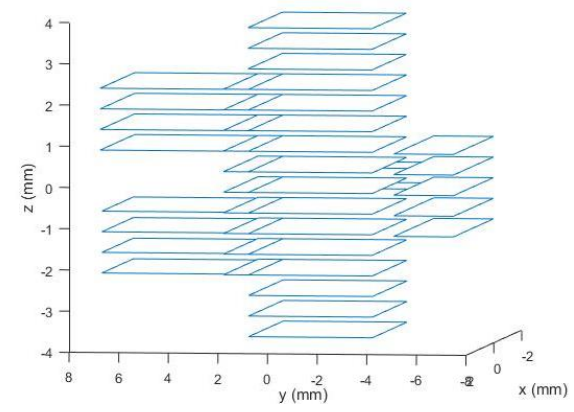

(a) SE function

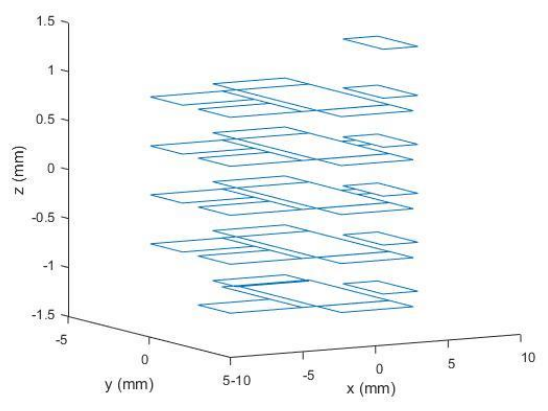

(b) SA function

Figure 6: Humanoid object optimal solution for both $S E$ and $S A$ functions.

by a rotation around the $y$ axis due to the object wing.

\section{Conclusions and future work}

In this work we presented a strategy to optimize the staircase effect and the need for supports generation of objects in 3D printing. While two apparently conflicting objective functions had been proposed in the literature for this purpose, we focused on this paper on symmetrical objects, showing that in this case these two objective functions are no longer conflicting.

Our strategy was then the application of a state-of-art optimization solver for global derivative-free optimization to the single minimization of the staircase effect and the supports generation. The proposed approach enables the computation of the optimal orientation of objects for 3D printing when the layer thickness is constant and there is some symmetry in the object. Our approach does not modify the original object geometry, and the optimal orientation obtained is not guaranteed to entirely remove the staircase effect or the need to support building.

We illustrated the proposed strategy with three objects. Two of them come from the aerospace industry, where object symmetry is present. The third one is a toy example providing an object without complete symmetry. The reported numerical results have shown the effectiveness and robustness of the proposed approach. Additionally, our numerical findings have confirmed the non conflicting nature of the two objective functions under symmetry of the objects.

As future work, we plan to integrate the developed optimization strategy into a 3D printer with 5-axes, leading to efficient printing with reduced staircase effect and the need of supports. We also plan to apply our optimization strategy to the 3D printing of real objects in the aerospace industry. Another extension of the current work could be to optimize the process parameters in rapid prototyping technologies, in particular the used layer thickness (which depends on the technology type) and the cost of the allowable overhanging parts without support structure. 


\section{Acknowledgements}

This work was developed under the FIBR3D project Hybrid processes based on additive manufacturing of composites with long or short fibers reinforced thermoplastic matrix (POCI-01-0145-FEDER-016414), supported by the Lisbon Regional Operational Programme 2020, under the PORTUGAL 2020 Partnership Agreement, through the European Regional Development Fund (ERDF).

Support was also given by the FCT project grants UID/MAT/00324/2013, and P2020 SAICTPAC/0011/2015.

\section{References}

[1] P. B. Bacchewar, S. K. Singhal, and P. M. Pandey. Statistical modelling and optimization of surface roughness in the selective laser sintering process. Proceedings of the Institution of Mechanical Engineers, Part B: Journal of Engineering Manufacture, 221(1):35-52, 2007.

[2] W. Cheng, J.Y.H. Fuh, A.Y.C. Nee, Y.S. Wong, H.T. Loh, and T. Miyazawa. Multiobjective optimization of part building orientation in stereolithography. Rapid Prototyping Journal, 1(4):12-23, 1995.

[3] V. Dhir, T. Itoi, P. Fockens, M. Perez-Miranda, M. A. Khashab, D. W. Seo, A. M. Yang, K. Yu Lawrence, and A. Maydeo. Novel ex vivo model for hands-on teaching of and training in eus-guided biliary drainage: creation of mumbai eus stereolithography/3d printing bile duct prototype (with videos). Gastrointestinal Endoscopy, 81(2):440-446, 2015.

[4] D. Ding, Z. Pan, D. Cuiuri, and H. Li. A practical path planning methodology for wire and arc additive manufacturing of thin-walled structures. Robotics and ComputerIntegrated Manufacturing, 34:8-19, 2015.

[5] D. Ding, Z. Pan, D. Cuiuri, H. Li, and N. Larkin. Adaptive path planning for wirefeed additive manufacturing using medial axis transformation. Journal of Cleaner Production, 133:942-952, 2016.

[6] A. Dolenc and I. Makela. Slicing procedures for layered manufacturing techniques. Computer-Aided Design, 26(2):119-126, 1994.

[7] L. P. C. Gomez, A. Spangenberg, X. Ton, Y. Fuchs, F. Bokeloh, J. Malval, B. Tse Sum Bui, D. Thuau, C. Ayela, K. Haupt, and O. Soppera. Rapid prototyping of chemical microsensors based on molecularly imprinted polymers synthesized by two-photon stereolithography. Advanced Materials, 28(28):5931-5937, 2016. 
[8] B. C. Gross, J. L. Erkal, S. Y. Lockwood, C. Chen, and D. M. Spence. Evaluation of $3 \mathrm{~d}$ printing and its potential impact on biotechnology and the chemical sciences. Analytical Chemistry, 86(7):3240-3253, 2014.

[9] T. Grünberger and R. Domröse. Direct metal laser sintering. Laser Technik Journal, 12(1):45-48, 2015.

[10] B. Huang and S. Singamneni. Curved layer adaptive slicing (clas) for fused deposition modelling. Rapid Prototyping Journal, 21(4):354-367, 2015.

[11] A. Hussein, L. Hao, C. Yan, R. Everson, and P. Young. Advanced lattice support structures for metal additive manufacturing. Journal of Materials Processing Technology, 213(7):1019-1026, 2013.

[12] Z. Jibin. Determination of optimal build orientation based on satisfactory degree theory for rpt. In Ninth International Conference on Computer Aided Design and Computer Graphics (CAD-CG'05), pages 6 pp.-, 2005.

[13] Y. Jin, J. Du, Y. He, and G. Fu. Modeling and process planning for curved layer fused deposition. The International Journal of Advanced Manufacturing Technology, pages $1-13,2016$.

[14] Y. Jin, J. Du, Z. Ma, A. Liu, and Y. He. An optimization approach for path planning of high-quality and uniform additive manufacturing. The International Journal of Advanced Manufacturing Technology, pages 1-12, 2017.

[15] J. Jung and R. S. Ahluwalia. Nc tool path generation for 5-axis machining of free formed surfaces. Journal of Intelligent Manufacturing, 16(1):115-127, 2005.

[16] M. W. Khaing, J.Y.H. Fuh, and L. Lu. Direct metal laser sintering for rapid tooling: processing and characterisation of EOS parts. Journal of Materials Processing Technology, 113(1-3):269-272, 2001.

[17] S. Kolossov, E. Boillat, R. Glardon, P. Fischer, and M. Locher. 3d FE simulation for temperature evolution in the selective laser sintering process. International Journal of Machine Tools and Manufacture, 44(2-3):117-123, 2004.

[18] P. Kulkarni, A. Marsan, and D. Dutta. A review of process planning techniques in layered manufacturing. Rapid Prototyping Journal, 6(1):18-35, 2000.

[19] A. Kumar, A. G. Banerjee, S. Paul, and A. R. Choudhury. Maximization of slice height with uniformity in surface roughness in the direct slicing of freeform surfaces. Proceedings of the Institution of Mechanical Engineers, Part B: Journal of Engineering Manufacture, 217(6):765-777, 2003.

[20] K. H. Lee and K. Choi. Generating optimal slice data for layered manufacturing. The International Journal of Advanced Manufacturing Technology, 16(4):277-284, 2000. 
[21] W. Lee, C. Wei, and S. Chung. Development of a hybrid rapid prototyping system using low-cost fused deposition modeling and five-axis machining. Journal of Materials Processing Technology, 214(11):2366-2374, 2014.

[22] H. Li, T. Wang, J. Sun, and Z. Yu. The adaptive slicing algorithm and its impact on the mechanical property and surface roughness of freeform extrusion parts. Virtual and Physical Prototyping, 11(1):27-39, 2016.

[23] S. Lim, R. A. Buswell, P. J. Valentine, D. Piker, S. A. Austin, and X. De Kestelier. Modelling curved-layered printing paths for fabricating large-scale construction components. Additive Manufacturing, 12, Part B:216-230, 2016.

[24] F. Ning, W. Cong, J. Qiu, J. Wei, and S. Wang. Additive manufacturing of carbon fiber reinforced thermoplastic composites using fused deposition modeling. Composites Part B: Engineering, 80:369-378, 2015.

[25] W. Oropallo and L. A. Piegl. Ten challenges in 3d printing. Engineering with Computers, 32(1):135-148, 2016.

[26] P. M. P., N. V. Reddy, and S. G. Dhande. Improvement of surface finish by staircase machining in fused deposition modeling. Journal of Materials Processing Technology, 132(1-3):323-331, 2003.

[27] S. S. Pande and S. Kumar. A generative process planning system for parts produced by rapid prototyping. International Journal of Production Research, 46(22):6431-6460, 2008.

[28] P. M. Pandey, N. V. Reddy, and S. G. Dhande. Real time adaptive slicing for fused deposition modelling. International Journal of Machine Tools and Manufacture, 43(1):61-71, 2003.

[29] P. M. Pandey, K. Thrimurthulu, and N. V. Reddy. Optimal part deposition orientation in fdm by using a multicriteria genetic algorithm. International Journal of Production Research, 42(19):4069-4089, 2004.

[30] B. Peters. Architectural Research Addressing Societal Challenges. Taylor \& Francis Group, 2017.

[31] R. Ponche, O. Kerbrat, P. Mognol, and J. Hascoet. A novel methodology of design for additive manufacturing applied to additive laser manufacturing process. Robotics and Computer-Integrated Manufacturing, 30(4):389-398, 2014.

[32] S. Rianmora and P. Koomsap. Recommended slicing positions for adaptive direct slicing by image processing technique. The International Journal of Advanced Manufacturing Technology, 46(9):1021-1033, 2010. 
[33] D. T. Richard and R. H. Crawford. Optimizing part quality with orientation. In The University of Texas at Austin, pages 362-368, 1995.

[34] A. Simchi, F. Petzoldt, and H. Pohl. On the development of direct metal laser sintering for rapid tooling. Journal of Materials Processing Technology, 141(3):319-328, 2003.

[35] S. K. Singhal, P. K. Jain, and P. M. Pandey. Adaptive slicing for sls prototyping. Computer-Aided Design and Applications, 5(1-4):412-423, 2008.

[36] G. Strano, L. Hao, R. M. Everson, and K. E. Evans. A new approach to the design and optimisation of support structures in additive manufacturing. The International Journal of Advanced Manufacturing Technology, 66(9):1247-1254, 2013.

[37] K. Thrimurthulu, P. M. Pandey, and N. V. Reddy. Optimum part deposition orientation in fused deposition modeling. International Journal of Machine Tools and Manufacture, 44(6):585-594, 2004.

[38] A. I. F. Vaz and L. N. Vicente. A particle swarm pattern search method for bound constrained global optimization. J. Global Optim., 39:197-219, 2007.

[39] A. I. F. Vaz and L. N. Vicente. Pswarm: A hybrid solver for linearly constrained global derivative-free optimization. Optim. Methods Softw., 24:669-685, 2009.

[40] W. Wang, H. Chao, J. Tong, Z. Yang, X. Tong, H. Li, X. Liu, and L. Liu. Saliencypreserving slicing optimization for effective 3d printing. Computer Graphics Forum, 34(6):148-160, 2015.

[41] J. M. Williams, A. Adewunmi, R. M. Schek, C. L. Flanagan, P. H. Krebsbach, S. E. Feinberg, S. J. Hollister, and S. Das. Bone tissue engineering using polycaprolactone scaffolds fabricated via selective laser sintering. Biomaterials, 26(23):4817-4827, 2005. 